\title{
Surface Plasmon Resonance Sensor to Detect n-Hexane in Palm Kernel Oil Using Polypyrrole Nanoparticles Reduced Graphene Oxide Layer
}

\author{
Amir Reza Sadrolhosseini ${ }^{D},{ }^{1}$ Mina Habibiasr, ${ }^{2}$ Hassan Soleimani, ${ }^{3}$ \\ Mohd Nizar Hamidon (D, ${ }^{1}$ Yap Wing Fen, ${ }^{1,4}$ and H. N. Lim ${ }^{5}$ \\ ${ }^{1}$ Functional Device Laboratory, Institute of Advanced Technology, Universiti Putra Malaysia, 43400UPM, Serdang, \\ Selangor, Malaysia \\ ${ }^{2}$ Department of Process and Food Engineering, Faculty of Engineering, Universiti Putra Malaysia, 43400UPM, Serdang, \\ Selangor, Malaysia \\ ${ }^{3}$ Department of Fundamental and Applied Science, Universiti Teknologi PETRONAS, 3175 Seri Iskandar, Malaysia \\ ${ }^{4}$ Department of Physics, Faculty of Science, University Putra Malaysia, 43400UPM Serdang, Selangor, Malaysia \\ ${ }^{5}$ Department of Chemistry, Faculty of Science, University Putra Malaysia, 43400UPM Serdang, Selangor, Malaysia
}

Correspondence should be addressed to Amir Reza Sadrolhosseini; amir17984818@gmail.com and Mohd Nizar Hamidon; mnh@upm.edu.my

Received 10 March 2020; Revised 14 September 2020; Accepted 7 October 2020; Published 4 January 2021

Academic Editor: Aitor Urrutia

Copyright (c) 2021 Amir Reza Sadrolhosseini et al. This is an open access article distributed under the Creative Commons Attribution License, which permits unrestricted use, distribution, and reproduction in any medium, provided the original work is properly cited.

Palm kernel oil was extracted using n-hexane. Rotary evaporator and oven were used to remove n-hexane from the oil. Measurement of $\mathrm{n}$-hexane in low concentration is significant and interest subject. In this study, the concentration of $\mathrm{n}$-hexane was measured using the surface plasmon resonance technique. In order to improve the sensitivity of surface plasmon resonance sensor, the polypyrrole nanoparticles decorated reduced graphene oxide (sensing layer) was prepared using the electrodeposition technique on the surface of gold film. Different concentration of n-hexane in isooctane and the palm kernel oil before and after purification was tested using sensing layer. The sensor limit was about $1 \mathrm{ppm}$. The results were matched with the gas chromatography results. The thickness and roughness of sensing layer were increased after the interaction with n-hexane which was obtained from atomic force microscopy.

\section{Introduction}

Palm kernel oil is rich in saturated fatty acids and a small amount of unsaturated fatty acids. Palm kernel oil is a white to yellowish oil which is solid at normal temperature and is considered a secondary product of palm fruit [1]. Palm kernel oil contains approximately $80 \%$ saturated fatty acid comprised of $\mathrm{C} 12$ or lauric acid (48.7\%), C14 or myristic acid (15.6\%), and C16 or palmitic acid (7.5\%). Unsaturated fatty acids are included C18:1 or oleic acid (14.8\%) and C18:2 or linoleic acid (2.6\%) [2]. Solvent extraction in organic solution is a popular method for palm kernel oil extraction [3]. Currently, $\mathrm{n}$-hexane is the preferred solvent throughout the world. n-Hexane has been categorized as a hazardous air pollutant (HAP) by the US Environmental Protection Agency, and it is included in the list of toxic chemicals [4]. According to PFA Act 1954, the maximum permissible limit for $\mathrm{n}$-hexane in oil and the meal are about $5 \mathrm{ppm}$ and $10 \mathrm{ppm}$, respectively [5]. Therefore, $\mathrm{n}$-hexane must be removed totally from palm kernel oil after extraction of oil from the kernel. Typically, rotary evaporator and oven are used to eliminate the $\mathrm{n}$-hexane from palm kernel oil. The analytical methods such as Fourier transform infrared spectroscopy (FT-IR) [6] and gas chromatography (GC) [7] are used to measure the concentration of $n$-hexane. The mentioned methods are complicated, and they depend on chemical 
knowledge, nonlinear calibration carves, and high cost instrument. Since the $\mathrm{n}$-hexane is a toxic chemical and the detection of $n$-hexane in edible oil is important, therefore, designing a simple sensor for measurement of low concentration of $\mathrm{n}$-hexane is vital.

The surface plasmon resonance (SPR) is a versatile and accurate technique to measure and detect the toxic chemical [8-11] and to verify the toxic chemical concentration in solution. Typically, the SPR signal is achieved from the interaction of the light beam with gold thin film in the interface of a metal and dielectric medium $[11,12]$. In order to increase the sensitivity and selectivity of sensor and biosensor, the gold layer should be modified by polymer and polymer nanocomposite materials to enhance the physical structure [13] and surface chemistry [14]. Labeled nanoparticles [15] are used to enhance the sensitivity of sensor and biosensor. Recently, the polymer composites based on the carbon based nanomaterial such as graphene [16], graphene oxide [17], graphene quantum dots [18], and reduced graphene oxide [19] were used to modify the metal layer for sensor application.

Polypyrrole (PPy) has intrinsic conducting polymer. PPy is used for some industrial application such as microelectronic device, sensor, and biosensor [20,21]. PPy has $\pi$-electron conjugation $[22,23]$ which is used to explain the electronic properties and high electron affinity [24]. Hence, $\mathrm{PPy}$ is considerable to prepare the sensing layer.

Graphene oxide (GO) can be derived from graphite. It is a two-dimensional structure, and it has a single layer atomic molecule with covalent $\mathrm{C}-\mathrm{O}$ bonds [25]. The main functional group of GO is the hydroxyl (OH-) and epoxy (-COO-) groups at the basal plane and at the edge of the molecular structure, respectively [26]. The reduce graphene oxide (RGO) will be achieved when the oxygen of functional group will be removed and the electronic properties will be enhanced [27]. GO and RGO have potential for sensor, biosensor [28], and detection of toxic chemical in the gas form such as $\mathrm{CO}_{2}$ [29]. Graphene and reduced graphene oxide have the strong nonlinearity [30], so they have potential in active optical device such as fiber laser [31] and optical detector. GO, RGO, and MXene [32] are the 2D nanostructure, and they are high potential candidate for SPR sensor as a sensing layer.

As was mentioned above, polypyrrole has high electron affinity and tendency to absorb the chemical material. The functional group of reduce graphene oxide can interact with chemical material. So, to enhance the sensitivity of the sensing layer, the composite of polypyrrole nanoparticles reduce graphene oxide was used to detect and measure the concentration of $\mathrm{n}$-hexane. Polypyrrole nanoparticles decorated reduce graphene oxide (PPy-NPs/RGO) was prepared using the electrodeposition technique to modify the gold layer. The PPy-NPs/RGO was applied to measure the low and high concentrations of $n$-hexane. Initially, the solution of $n$ hexane in isooctane was used to investigate the interaction of $\mathrm{n}$-hexane with sensing layer. The sensing layer was applied to measure the $n$-hexane in palm kernel oil using the SPR technique. The results were compared with the gas chromatography results. The sensing layer was tested before and after the sensing of the n-hexane using atomic force microscopy (AFM).

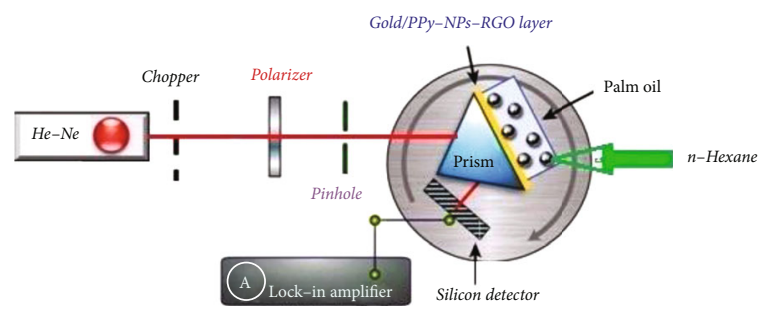

Figure 1: The SPR setup to measure the low concentration of n-hexane. The setup contains a $\mathrm{He}-\mathrm{Ne}$ laser, a chopper, a polarizer, a pinhole, a prism, a fluid tank, and a lock in amplifier.

\section{Materials and Methods}

2.1. Materials. The chemical materials such as $\mathrm{n}$-hexane, isooctane, $\mathrm{FeCl}_{3}$, sodium para-toluene sulfonate, and pyrrole were purchased from Sigma-Aldrich Company.

2.2. Preparation of Palm Kernel Oil. Palm kernels were obtained from palm kernel mill at Carey Island, Malaysia. Palm kernels were sieved manually to remove the foreign material, broken kernels, shell, fiber, nut, and stones.

Palm kernel oil was extracted from palm kernel with different moisture contents of $28 \%, 17 \%$, and $7 \%$. Different moisture content of palm kernel was obtained by drying of palm kernel. Palm kernel oil was extracted using n-hexane [33]. Prior to oil extraction, the kernels were grinded, and $100 \mathrm{~g}$ of kernel powder was soaked in $200 \mathrm{~mL}$-hexane for $48 \mathrm{~h}$ with frequent shaking. The mixture was filtered, and $\mathrm{n}$-hexane was separated from the oil by a rotary evaporator (IKA, RV 10 digital, German). Extracted oils were kept in different Pyrex glass breaker and were put inside an oven at $103^{\circ} \mathrm{C}$. The samples were removed from the oven every $2 \mathrm{~h}$ and were used to test the surface plasmon resonance sensor using polypyrrole decorated reduce graphene oxide to measure the concentration of $n$-hexane. The palm kernel oil was characterized using Fourier transform infrared spectroscopy (FT-IR) and gas chromatography (GC).

2.3. Gas Chromatography. A gas chromatograph (Model 5890; Hewlett-Packard, Palo Alto, CA) with a FID (Hewlett-Packard model 3392 A integrator) and a BPX70 polar capillary column (SGE, Victoria, Australia; $0.32 \mathrm{~mm}$ i.d., $30 \mathrm{~m}$ length; $0.25 \mu \mathrm{m}$ film thickness) was used to measure the concentration of $n$-hexane in prepared samples. The carrier gas was the pure helium gas $(99.99 \%, 23 \mathrm{~mL} / \mathrm{min})$, and the $\mathrm{n}$-hexane peak was recognized by comparison with the survival time of the valid standard. The amounts of $n$ hexane were calculated from the arose area peak in different concentration of $\mathrm{n}$-hexane in standard samples [5].

2.4. Sensing Layer. The gold layer was prepared on the surface of a microscope glass slide using sputtering coating in $49.2 \mathrm{~nm}$. After that, PPy-NPs/RGO was deposited on the surface of gold coated glass slide using electrodeposition technique $[9,34]$. GO was synthesized using the simplified Hummer's method [35] which was used to reduce the graphene oxide precursor for the formation of the PPyNPs/RGO nanocomposite layer. The layer was synthesized 

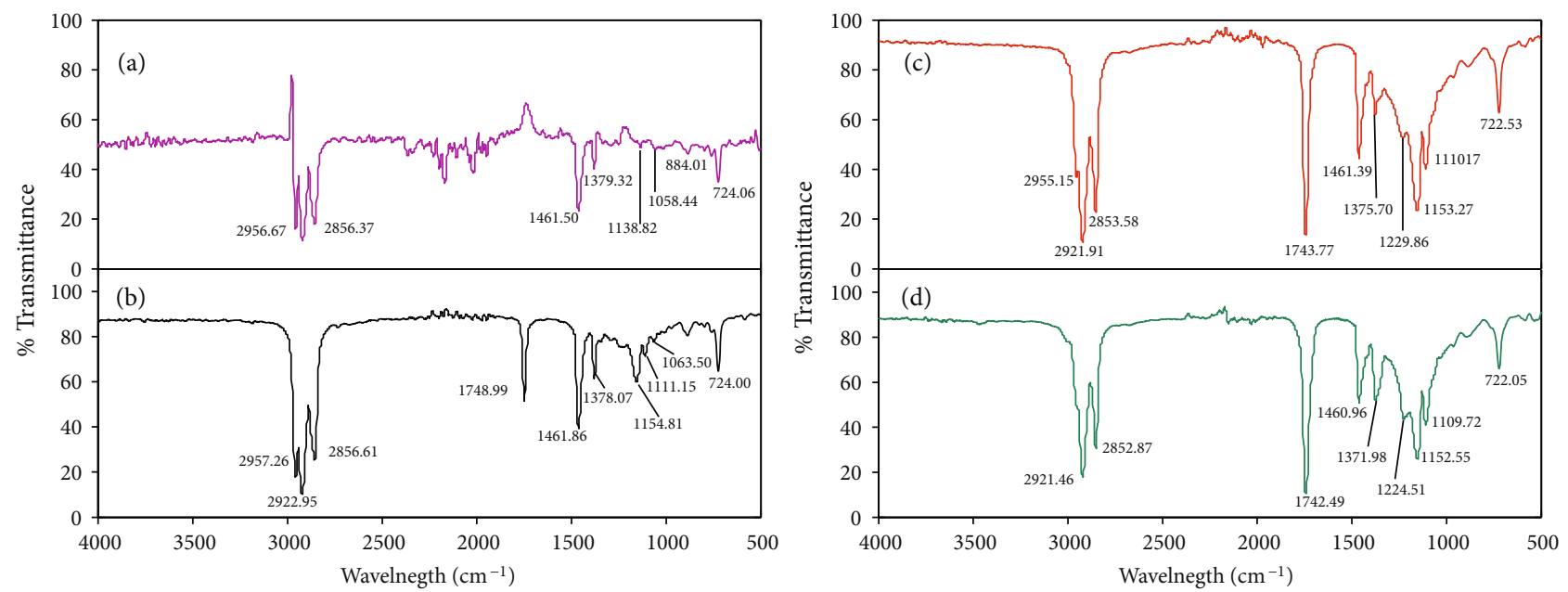

FIGURE 2: The FT-IR results show the functional group of (a) pure n-hexane (b) palm kernel oil before evaporation in the rotary evaporator, (c) palm kernel oil after evaporation in a rotary evaporator, and (d) palm kernel oil after evaporation in the oven.

by electro-polymerization from a solution that contained $0.1 \mathrm{M}$ pyrrole, $1 \mathrm{mg} / \mathrm{mL} \mathrm{GO}, 0.1 \mathrm{M}$ sodium para-toluene sulfonate (Nap TS), and $1.0 \mathrm{mM} \mathrm{FeCl}_{3}$. The layers were deposited on a glass slide with coated gold layer using a potentiostat-galvanostatic (Elchema model EQCN-502 Faraday cage). A graphite electrode was used as the counter electrode, and the working electrode was glass slide coated with gold layer at a thickness of $42 \mathrm{~nm}$. All of the potentials were referred to a saturated calomel electrode (SCE). The time of polymerization was $25 \mathrm{~min}$. The thickness and refractive index of the layer were obtained using a high surface profilometer (AMBIOS Technology XP-200) with the limitation of about $\pm 8 \mathrm{~nm}$ and surface plasmon resonance technique.

2.5. Surface Plasmon Resonance Setup. Figure 1 shows the SPR setup based on the Keretchman configuration. The SPR setup contains a He-Ne laser, a chopper, a pinhole, a rotation stage, a high index prism, a fluid tank, a silicon detector, and a lock-in amplifier [36, 37]. The high refractive index prism was placed on the rotation stage bench. The gold layer and PPy-NPs/RGO were prepared prior of doing the experiment on the microscope glass slide. The microscope glass slide with sensing layer was attached to the prism using index matching gel (F-IMF-105, Newport, USA). The sample was injected to the fluid tank. The different concentration of $\mathrm{n}$-hexane in palm kernel oil was separately contacted to sensing layer using a fluid tank. The experiment was carried out using the static regime. The laser beam with $6 \mathrm{~mm}$ spot size passed through the prism, and the intensity of the laser beam was registered when the rotation stage stopped momently. The rotation angle was increased up to $30^{\circ}$. The SPR signal was registered in the room temperature.

The refractive index and thickness of the gold layer are equal to $0.236+3.31 i$ and $49.2 \mathrm{~nm}$, respectively. At first, the experiment was carried out to measure the refractive index of PPy-NPs/RGO. After that, the sensing layer was used to investigate the SPR signal to detect the $n$-hexane in isooctane because isooctane is usually used as a standard solvent. In the end, the sensing layer was used to detect and measure the $n$ hexane in the palm kernel oil.

In order to analyze the SPR signal, the Fresnel theory and Fresnel formula for n-layer were used to analyze the SPR signals. The intensity of reflected laser beam should be registered to obtain the SPR signals. Hence, the reflection coefficient derived from the Fresnel theory [11] should be considered to analyze the SPR signals. Usually, the gold layer was sandwiched between the high refractive index prism and the dielectric solution, and the incident wave is in the $y-z$ plan. Hence, the amplitude of the reflected laser beam $\left(A_{r}\right)$ could be written as:

$$
A_{r}=r_{123} A_{i}
$$

where $A_{i}$ is the amplitude of the incident light and $r_{123}$ represents the amplitude reflection coefficient which relies on thickness $(t)$ as given below:

$$
r_{123}=\frac{r_{12}+r_{23} \exp \left(2 i k_{2 x} t\right)}{1+r_{12} r_{23} \exp \left(2 i k_{2 x} t\right)},
$$

where $r_{12}, r_{23}$, and $k_{2 x}$ are the reflection coefficient, glass metal reflection coefficient, and phase constant, respectively $[11,12,28]$, and the $k_{i x}$ and $k_{j x}$ can be defined as.

$$
k_{i x}=\sqrt{\left(\frac{2 \pi}{\lambda}\right)^{2} \varepsilon_{i}-k_{z}^{2}}, k_{j x}=\sqrt{\left(\frac{2 \pi}{\lambda}\right)^{2} \varepsilon_{j}-k_{z}^{2}},
$$

where the $k_{z}$ is the propagation constant $\left((2 \pi / \lambda) n_{p} \sin \theta_{2}\right)$ and $r_{i j}$ is

$$
r_{i j}=\frac{\varepsilon_{j} k_{i x}-\varepsilon_{i} k_{j x}}{\varepsilon_{j} k_{i x}+\varepsilon_{i} k_{j x}}, i, j=1,2,3,
$$

where $\varepsilon_{j}$ and $\varepsilon_{i}$ are the dielectric constant. The reflectance 


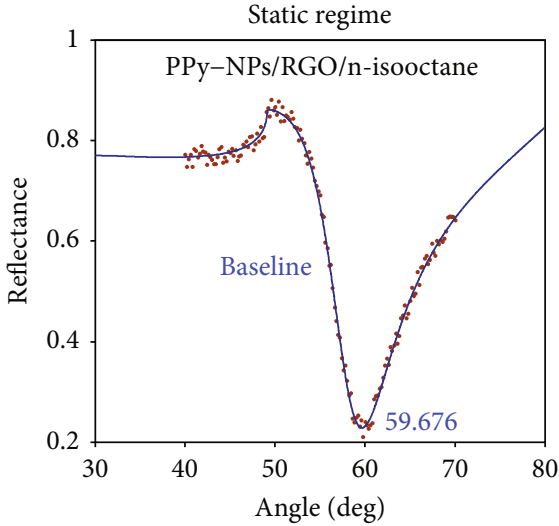

(a)

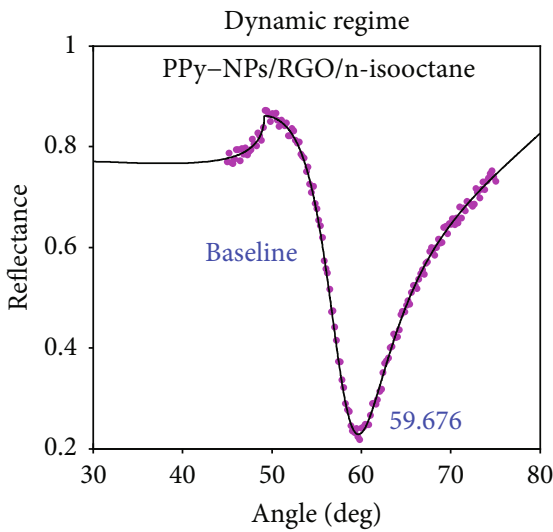

(b)

FIgURe 3: The SPR signal to determine the refractive index of PPy-NPs/RGO and the baseline in the presence of isooctane in (a) static regime, (b) dynamic regime.

is $R=\left|r_{123}\right|^{2}$, and it is a function of dielectric constant, thickness of layer, and resonance angle.

The refractive index of gold layer and the sample can be found by the resonance condition $[11,12]$ as follows:

$$
n_{p} \sin \theta_{R}=\sqrt{\frac{\left(n_{1}^{2} n_{2}^{2}\right)}{\left(n_{1}^{2}+n_{2}^{2}\right)}},
$$

where $\theta_{R}, n_{p}, n_{1}$, and $n_{2}$ are the resonance angle, refractive index of prism, refractive index of gold layer, and the sample, respectively. The refractive index of the sample is

$$
n_{2}=\sqrt{\frac{\left(n_{1}^{2} n_{p}^{2} \sin ^{2} \theta_{R}\right)}{\left(n_{1}^{2}-n_{p}^{2} \sin ^{2} \theta_{R}\right)}},
$$

when the sensing layer was used to detect the chemical material, and the SPR configuration contains the high refractive index prism/gold layer/sensing layer/prob medium. Hence, Eq. (1) was improved for many layers that were discussed in Ref. $[8,11]$ to find the resonance angle and refractive index. Therefore, reflectance $(R)$ is a function of optical properties of the metal layer, sensing layer, prob medium, and prism. When the thickness of the gold layer and refractive index of the gold layer are known, the optical properties of the sensing layer and dielectric solution can be obtained by minimizing following summation $[28,38]$ :

$$
\Gamma=\sum_{\theta}\left[R_{\operatorname{Exp}}\left(\theta_{2}, n_{2}\right)-R_{\text {Theory }}\left(\theta_{2}, n_{2}\right)\right],
$$

where $R_{\text {Exp }}$ and $R_{\text {Theory }}$ are the experimental and theoretical reflectance, respectively. The reflectance is a function of angle refractive index and wavelength.

In this study, the SPR configuration such as high index prism/gold layer/PPy-NPs-RGO/water was used to determine the refractive index of PPy-NPs/RGO, and the SPR configuration contains high index prism/gold layer/n-hexane solution was used to determine the refractive index of $n$ -

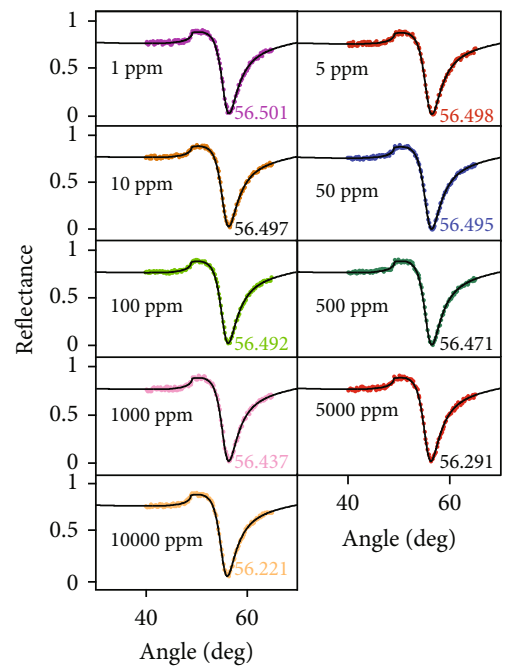

FIgURE 4: The experiment was carried out with the prism/gold layer system to determine the refractive index of $n$-hexane solution in different concentration. The SPR signals were registered, and the resonance angle was in the range of $56.501^{\circ}$ to $56.221^{\circ}$.

hexane solution based on Eq. (5). After that, the SPR configuration contains high index prism/gold layer/PPy-NPs-RGO (sensing layer)/probe sample was used to detect the nhexane. The refractive indices of the sensing layer and nhexane solution were used to analyze the SPR signals to obtain the variation of resonance angle with time. The SPR signal was analyzed using the computer program based on a minimum root square to obtain the resonance angle and resonance angle shift $[36,37]$.

2.6. Standard Sample. The pure palm kernel oil (Malaysian Palm Oil Board Product) was purchased. The h-hexane was added to $20 \mathrm{~mL}$ of pure palm kernel oil to prepare the solution of palm kernel oil with n-hexane in 1000 ppm concentration. The procedure was continued to find the lower concentration in 500,100, and 50 ppm for obtaining the standard samples. 
TABLE 1: The resonance angle and refractive index of n-hexane solution.

\begin{tabular}{|c|c|c|c|c|c|}
\hline Sample (ppm) & Angle of resonance & Refractive index n-hexane (SPR method) & $\Delta \theta_{\text {sat }}$ & $R^{2}(\%)$ & Refractive index n-hexane (Abbe method) \\
\hline 1 & 56.501 & 1.39094 & 0.014 & 95.0 & $1.3909 \pm 0.0002$ \\
\hline 5 & 56.498 & 1.39091 & 0.054 & 93.6 & $1.3908 \pm 0.0002$ \\
\hline 10 & 56.497 & 1.39089 & 0.112 & 96.0 & $1.3907 \pm 0.0002$ \\
\hline 50 & 56.495 & 1.39086 & 0.526 & 98.5 & $1.3908 \pm 0.0002$ \\
\hline 100 & 56.492 & 1.39083 & 0.965 & 97.2 & $1.3909 \pm 0.0002$ \\
\hline 500 & 56.471 & 1.39053 & 2.921 & 95.1 & $1.3906 \pm 0.0002$ \\
\hline 1000 & 56.437 & 1.39008 & 3.650 & 93.3 & $1.3901 \pm 0.0002$ \\
\hline 5000 & 56.291 & 1.38808 & 5.034 & 96.4 & $1.3882 \pm 0.0002$ \\
\hline 10000 & 56.221 & 1.38712 & 5.443 & 98.2 & $1.3890 \pm 0.0002$ \\
\hline
\end{tabular}
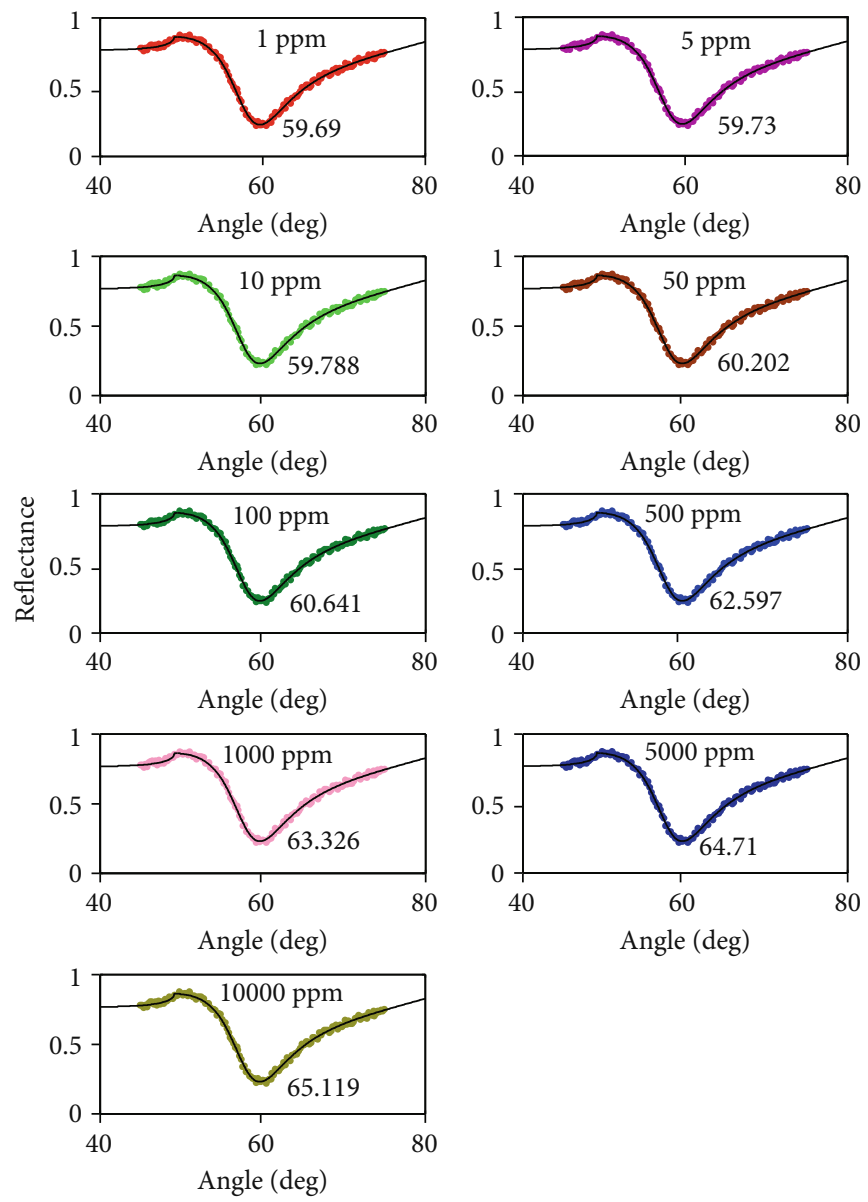

FIGURE 5: The SPR signals at the saturation value to obtain the variation of resonance angle with time and draw the sensogram for $n$-hexane in isooctane solution.

\section{Results and Discussion}

Figure 2 shows the FT-IR spectrum of pure hexane and palm kernel oil extracted. The spectrum 2(a) depicts the FT-IR related to pure hexane, and the main peaks appeared at 2956.676, 2920.55, 2856.37, 1461.50, 1379.32, 1138.82, $1058.44,884.01$, and $724.06 \mathrm{~cm}^{-1}$. The peaks related to $\mathrm{C}-\mathrm{H}$ and $\mathrm{O}-\mathrm{H}$ stretching occurred at 2956.676, 2920.55, and $2856.37 \mathrm{~cm}^{-1}$, and the peaks related to $\mathrm{C}=\mathrm{C}$ and $\mathrm{C}-\mathrm{H}$ binding emerged at 1461.50 and $1379.32 \mathrm{~cm}^{-1}$. The peak at 1058.44 , 884.01 , and $724.06 \mathrm{~cm}^{-1}$ represented the $\mathrm{C}-\mathrm{O}$ stretching and $=\mathrm{C}-\mathrm{H}$ bending [39].

The spectra 2(b), 2(c), and 2(d) related to palm kernel oil before evaporation in the rotary evaporator, palm kernel oil 


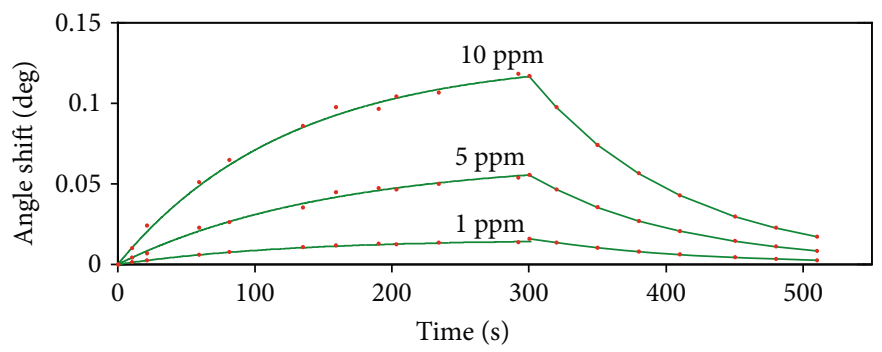

(a)

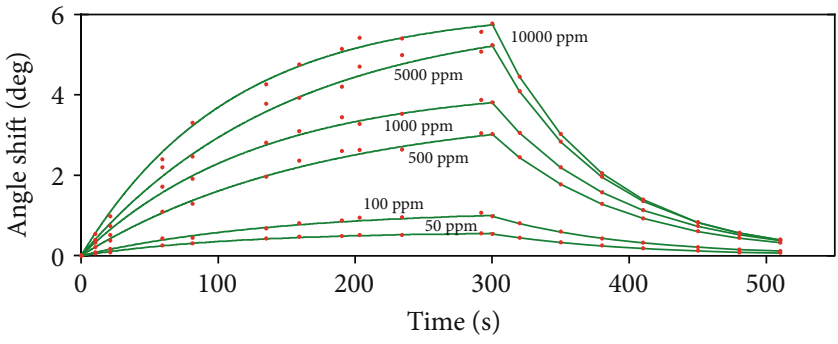

(b)

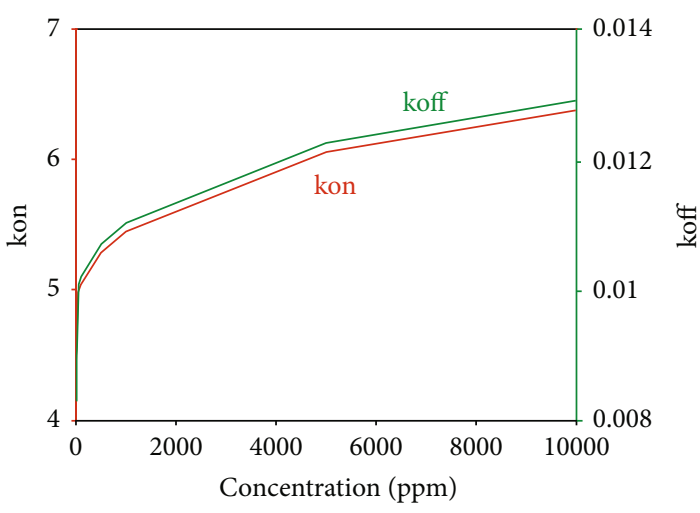

(c)

Figure 6: The sensograms $(\mathrm{a}, \mathrm{b})$ show the variation of resonance angle with time to detect the n-hexane in the isooctane solution. The association and dissociation process occurred from 0 to $300 \mathrm{~s}$ and $300 \mathrm{~s}$ to $510 \mathrm{~s}$, respectively. (c) Variation of $k_{\text {on }}$ and $k_{\text {off }}$ with the concentration of $n$-hexane.

TABLE 2: The pertinent value of resonance angle, resonance angle shift in saturation value, and $k_{\text {off }}$ and $k_{\text {on }}$ for different concentration of n-hexane dissolved in isooctane.

\begin{tabular}{|c|c|c|c|c|}
\hline Sample (ppm) & Angle of resonance at saturation & $\Delta \theta_{\text {sat }}$ & $k_{\text {off }}$ & $k_{\text {on }}$ \\
\hline 1 & $59.690^{\circ}$ & 0.014 & 0.0083 & 4.08465 \\
\hline 5 & $59.730^{\circ}$ & 0.054 & 0.0091 & 4.43406 \\
\hline 10 & $59.788^{\circ}$ & 0.112 & 0.0092 & 4.48327 \\
\hline 50 & $60.202^{\circ}$ & 0.526 & 0.0102 & 4.97539 \\
\hline 100 & $60.641^{\circ}$ & 0.965 & 0.0103 & 5.03445 \\
\hline 500 & $62.597^{\circ}$ & 2.921 & 0.0107 & 5.28052 \\
\hline 1000 & $63.326^{\circ}$ & 3.65 & 0.0111 & 5.44292 \\
\hline 5000 & $64.710^{\circ}$ & 5.034 & 0.0123 & 6.05315 \\
\hline 10000 & $65.119^{\circ}$ & 5.443 & 0.0129 & 6.37303 \\
\hline
\end{tabular}

after evaporation in a rotary evaporator, and palm kernel oil after evaporation in the oven. The main peaks at 2957.26, $2955.15,2922.95,2921.91,2921.46,2856.61,2853.58$, and $2852.87 \mathrm{~cm}^{-1}$ correspond to $-\mathrm{C}-\mathrm{H}$ stretching in long chain alkyl. The peaks at $1748.99,1743.77$, and $1742.49 \mathrm{~cm}^{-1}$ related to $\mathrm{C}=\mathrm{O}$ stretching and the peaks at 1461.86, 1461.39 , and $1460.96 \mathrm{~cm}^{-1}$ also depict the $\mathrm{C}=\mathrm{O}$ and $\mathrm{C}-\mathrm{O}=\mathrm{H}$ bending of fatty acids. There are peaks at 1229.86 and $1224.51 \mathrm{~cm}^{-1}$ represented the $-\mathrm{C}-\mathrm{N}$ stretching [35]. The peaks at $1378.07,1375.70$, and $1370.98 \mathrm{~cm}^{-1}$ related to $\mathrm{C}-\mathrm{N}$ stretching, and the peaks at $1154.81,1153.27$, and $1152.55 \mathrm{~cm}^{-1}$ related to $\mathrm{C}-\mathrm{O}$ stretching. The peaks which arose at $1111.15,1110.17$, and $1109.72 \mathrm{~cm}^{-1}$ authenticated the $\mathrm{C}-\mathrm{O}$ stretching of fatty acids. In addition, the peaks that appeared at $724.00,722.53 \mathrm{~cm}^{-1}$, and $722.05 \mathrm{~cm}^{-1}$ correspond to $=\mathrm{C}-\mathrm{H}$ out of plan bending [40].

Figure 2(b) shows the FT-IR spectrum for a mixture of nhexane and extracted palm kernel oil, since the FT-IT spectrum of n-hexane and palm kernel oil overlapped together. But the peaks at $1748.99 \mathrm{~cm}^{-1}$ related to palm kernel oil, and the peaks at 2957.26 and $1063 \mathrm{~cm}^{-1}$ confirmed the concentration of n-hexane in the mixture. Figures 2(c) and 2(d) show the FT-IR spectrum after evaporation and evaporation in the oven (heating). Figure 2(c) shows the intensity peaks at $2955.15 \mathrm{~cm}^{-1}$ decreased, and this peak disappeared in spectrum 2(d). Moreover, spectra 2(c) and 2(d) depict 


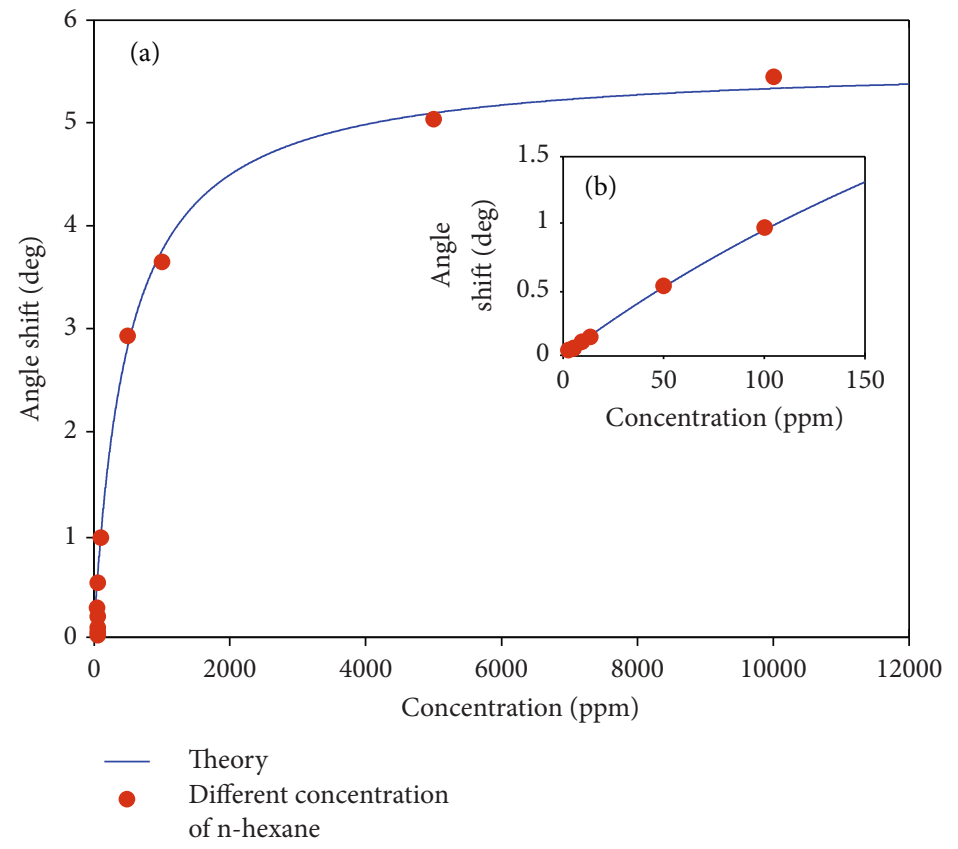

FIGURE 7: Calibration curve shows the variation of resonance angle shift with (a) low concentration and (b) high concentration of n-hexane.

the intensity peak at 1743.7 and $1742.49 \mathrm{~cm}^{-1}$ increased. Consequently, the extracted palm kernel oil contains the $\mathrm{n}$ hexane before evaporation, and the $\mathrm{n}$-hexane exists in palm kernel oil after evaporation.

The PPy-NPs/RGO was prepared using the electrodeposition method on the surface of the glass slide gold layer coated. The thickness of PPy-NPs/RGO was measured using a profilometer of about $10.3 \mathrm{~nm}$. In order to find the resonance angle at the baseline and the refractive index of layer, the PPy-NPs/RGO composite layer was attached to the high index prism $(n=1.83956)$, and the SPR signal was registered in the presence of pure isooctane $(n=1.391)$ at the room temperature $\left(21.5^{\circ} \mathrm{C}\right)$, and the SPR signal was stable in the room temperature (see Supplementary (available here)). The experiment was carried out in the static regime and dynamic regime. Figures 3(a) and 3(b) show the SPR signal in the static and dynamic regime, respectively. The resonance angle and refractive index of the sensing layer were obtained from the analysis the SPR signal using Fresnel Eqs. (1), (3), and (6) in the static and dynamic regime. Therefore, the resonance angle occurred at $59.676^{\circ}$, and the refractive index of PPy-NPs/RGO was $1.7261+i 0.241$. As a comparison of the static and dynamic regimes, the resonance angle and the refractive index are the same. Hence, the experiments were carried out at the static regime, and the baseline is $59.676^{\circ}$.

The different concentrations $(1,10,50,500,1000,5000$, and $10000 \mathrm{ppm}$ ) of $\mathrm{n}$-hexane were prepared in the isooctane solution, and they were separately contacted to the gold layer to obtain the refractive index of the solution. The SPR signals (Figure 4) were registered for each n-hexane solution, and the resonance angle and the refractive index of each n-hexane solution were achieved using the Fresnel equations (1), (3), (5) and (6). Moreover, the n-hexane solutions with different concentration were tested using the Abbe refractometer (Atago, NAR-1 T), and the results were compared with the
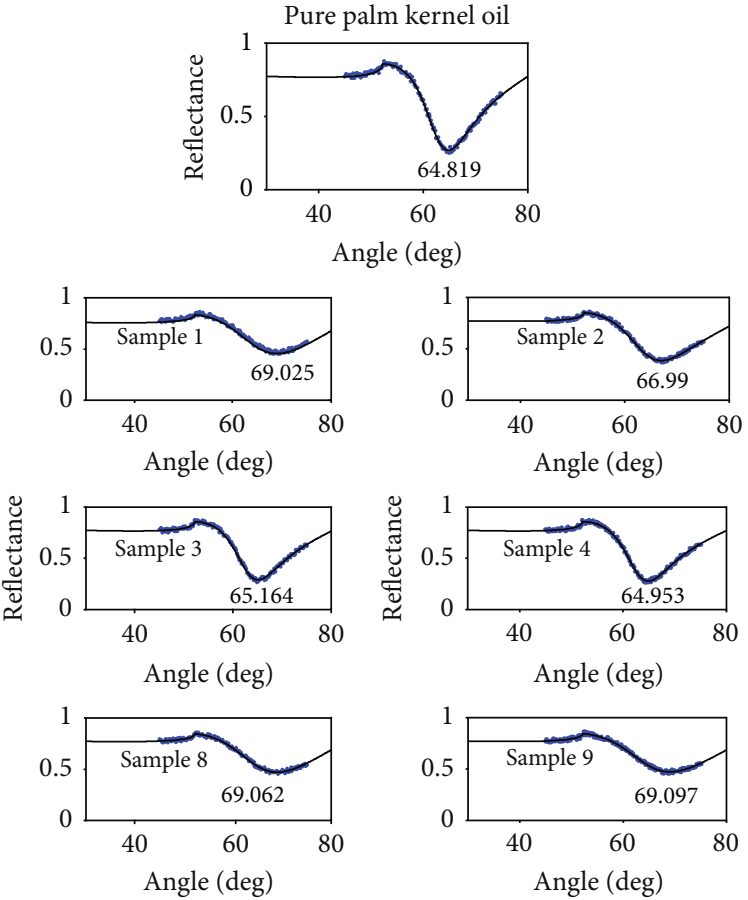

FIGURE 8: The SPR signals related to palm kernel oil at baseline and the SPR signals for tested sample (samples 1, 2, 3, 4, 5, 6, 7, 8, and 9) at saturation value to measure the concentration of $n$-hexane in palm kernel oil.

achieved refractive index from the SPR technique in Table 1. Consequently, they were matched together, and refractometry method confirmed the results that achieved from the SPR method.

The experiment was continued to detect the n-hexane and investigate the effect of the interaction of n-hexane with 


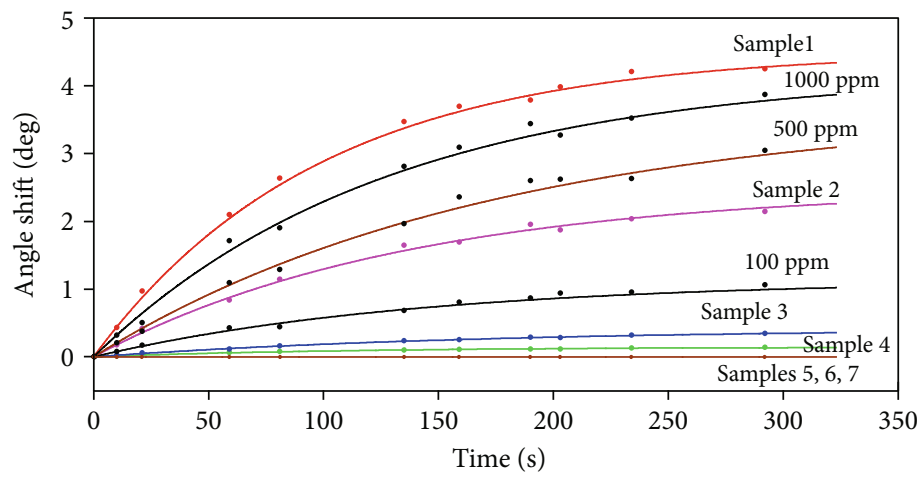

(a)

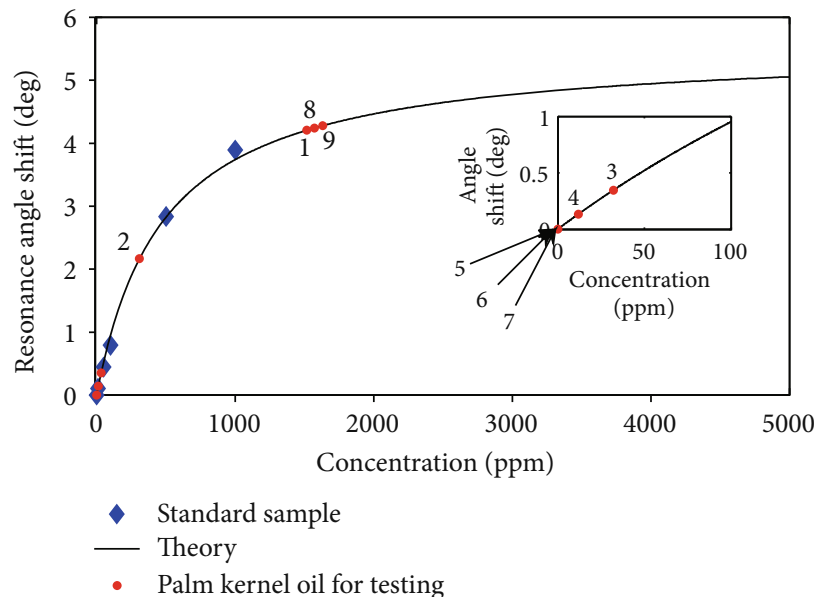

(b)

Figure 9: (a) The variation of resonance angle shift with time (standard sensogram) related to standard sample and test samples, (b) calibration curve to determine the concentration of $n$-hexane. The blue dots (blue diamond) and the red dots (red circle) are the value of resonance angle shift at saturation value which were derived from (a). Equation (9) was fitted to blue dots, and the red dots were used to find the concentration of the tested sample.

TABLE 3: The resonance angle shift and comparison of the concentration of n-hexane in palm kernel oil from the SPR sensor and GC method.

\begin{tabular}{|c|c|c|c|c|c|}
\hline Sample & Angle shift & Concentration of $\mathrm{n}$-hexane (SPR sensor) ppm & Concentration of $\mathrm{n}$-hexane (GC) ppm & $\Delta C$ & $R^{2}(\%)$ \\
\hline 1 & 4.206 & 1516 & 1514.7 & 2.7 & 93.0 \\
\hline 2 & 2.171 & 310 & 315 & 5 & 94.3 \\
\hline 3 & 0.345 & 32 & 29 & 3 & 97.0 \\
\hline 4 & 0.134 & 12 & 14 & 2 & 96.5 \\
\hline 5 & 0 & 0 & 0 & 0 & \\
\hline 6 & 0 & 0 & 0 & 0 & \\
\hline 7 & 0 & 0 & 0 & 0 & \\
\hline 8 & 4.243 & 1573 & - & - & 95.7 \\
\hline 9 & 4.278 & 1630 & - & - & 96.3 \\
\hline
\end{tabular}

PPy-NPs/RGO. So, the SPR signals were separately registered for each $n$-hexane solution, and the SPR signals were analyzed using Fresnel's equations (1), (3), and (5)-(7). The experiment was repeated 10 times to obtain the variation of resonance angle shift with time. Figure 5 shows SPR signal at saturation value, and Figure 6 shows the sensogram that obtained from subtracting the resonance angle at each time from resonance angle at baseline $\left(59.676^{\circ}\right)$.
The results were analyzed using the Langmuir's first order formula to explain the adsorption model as follows $[11,36,37]$ :

$$
\Delta \theta=\Delta \theta_{\text {sat }}\left(1-\exp \left(-k_{a} t\right)\right)
$$

where $\Delta \theta, \Delta \theta_{\text {sat }}, k_{a}$, and $t$ are resonance angle shift, resonance angle shift in saturation value, rate constant, and time, respectively. 
Figure 6 shows the sensogram of $n$-hexane detection. The dotted points are the experimental value, and they authenticated that the variation of resonance angle shift is a function of time. As a result, the experimental value is fitted well to the Langmuir's first order formula (solid line). The maximum value of each sensogram is equal to the saturation value that the SPR signals related to them have been presented in Figure 5. Moreover, Figure 6(a) shows the resonance angle shift with time for 10,5 , and $1 \mathrm{ppm}$ concentration of $\mathrm{n}$ hexane. Consequently, the minimum detection of $n$-hexane is $1 \mathrm{ppm}$.

Figures 6(a) and 6(b) also show the association and dissociation process. As a result, the $\mathrm{n}$-hexane released the sensing layer (PPy-NPs/RGO) with time as follows:

$$
\Delta \theta=\Delta \theta^{\prime} \exp \left(-k_{\text {off }} t\right)
$$

where $\Delta \theta^{\prime}$ and $K_{D}$ are the minimum resonance angle shift and the ration of $k_{\text {off }}$ and $k_{\text {on }}$, respectively. $k_{\text {on }}$ and $k_{\text {off }}$ are the second order rate constant of binding of $n$-hexane to sensing layer in $\mathrm{M}^{-1} \mathrm{~s}^{-1}$ unit and first order rate constant related to the dissociation of $\mathrm{n}$-hexane from sensing layer in $\mathrm{s}^{-1}$ unit, respectively, and they are a function of concentration. Hence, the variations of $k_{\text {off }}$ and $k_{\text {on }}$ with concentration of n-hexane have been presented in Figure 6(c), and the pertinent values have been listed in Table 2. Figures 6(a) and 6(b) confirm that the sensing layer is multiple use. The minimum concentration of $\mathrm{n}$-hexane that could be detected was about $1 \mathrm{ppm}$, and the resonance angle shift was about $0.028^{\circ}$ which was derived from sensogram (Figure 6(a)). The experiment was repeated more times to draw each sensogram, and the average of resonance angle shift for $1 \mathrm{ppm}$ was 0.027833 at saturation value, and the standard deviation was 0.0012133 . The limit of detection (LOD) defines as a minimum sensitivity of resonance angle shift. In accordance with the literature, LOD ( $\mathrm{LOD}=3 *(\sigma / S)$ ) is 3 times larger than ration of standard deviation $(\sigma)$ and sensitivity $(S)$. Hence, the LOD of the sensor is about $0.130^{\circ}$.

Figure 7 (calibration curve) shows the variation of the resonance angle shift in the saturation value with concentration of $n$-hexane in isooctane solution. That confirms, the variation of resonance angle shift increased with increasing the concentration of $n$-hexane, and the results fitted well to the Langmuir formula as follows [37, 41]:

$$
\Delta \theta_{\text {sat }}=\frac{\Delta \theta_{\max } \times C}{\left(1 / K_{D}\right)+C}
$$

where $\Delta \theta_{\max }, C$, and $K_{D}$ are the maximum of resonance angle shift, concentration of $\mathrm{n}$-hexane, and the affinity constant of n-hexane to sensing layer [42]. The values of $\Delta \theta_{\max }$ and $K_{D}$ are $5.595^{\circ}$ and 0.002032 , respectively.

The palm kernel oils were tested using SPR sensor based on PPy-NPs/RGO. Palm kernel oil was extracted using nhexane. Some portion of $n$-hexane was removed using rotary evaporator and oven. The oil was heated about $8 \mathrm{~h}$, and it was tested after every 2 hours to measure the concentration of $n$ hexane using the SPR sensor based on PPy-NPs/RGO thin

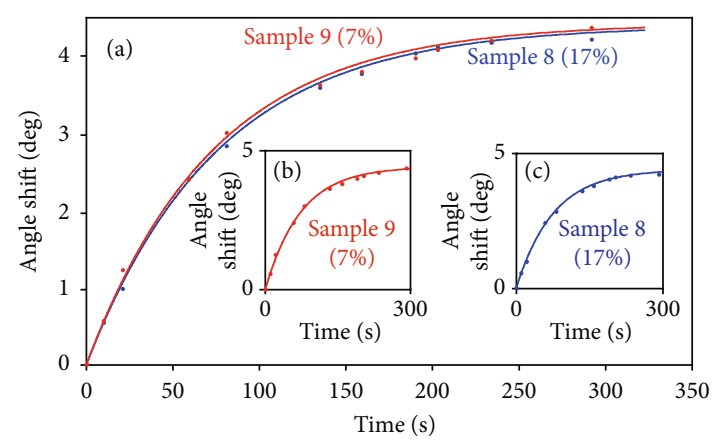

Figure 10: (a) The difference between variation of resonance angle shift with time (sensogram) for palm kernel oil with $7 \%$ and $17 \%$ moisture content, (b) sensogram of sample 9 with $7 \%$ moisture content, and (c) sensogram of sample 8 with $17 \%$ moisture content. Part (a) shows that the sensogram of sample 9 is upper than sensogram sample 8 .

layer. At the first step, the experiment was carried out using pure palm kernel oil and the standard samples (palm kernel oil $+n$-hexane 1000, 500,100, and $50 \mathrm{ppm}$ ). Therefore, the SPR signals of pure palm kernel oil were registered to obtain the baseline at $64.819^{\circ}$. The experiment was continued to obtain the standard sensogram. So, the standard samples (palm kernel oil $+n$-hexane 1000, 500, 100, and 50 ppm) were contacted with the sensing layer and registered the SPR signals. The SPR signals were analyzed based on the Fresnel equations (1), (3), and (6) to obtain the resonance angle and the resonance angle shift. The experiment was repeated 10 times to find the variation of resonance angle shift with time (standard sensogram). At the second step, the test samples (samples 1, 2, 3, 4, 5, 6, 7, 8, and 9) were separately contacted on the surface of sensing layer to register the SPR signals and obtain the variation of the resonance angle with time. For this propose, the experiment was also continued for 10 times. Figure 8 just shows the SPR signals for pure palm kernel oil and the SPR signals in the saturation value for each sample. Figure 9(a) depicts the standard sensogram for standard sample $(1000,500$, and $100 \mathrm{ppm})$ and tested sample (samples 1, 2, 3, 4, 5, 6, 7, 8, and 9). Figure 9(b) shows the variation of resonance angle shift with the concentration of $\mathrm{n}$-hexane for standard sample (blue dots). Equation (9) was used to analyze the data, and the solid line was achieved as a calibration curve. At the final step, the value of the resonance angle shift at saturation value for the test sample was used to find the concentration of samples (samples 1, 2, 3, $4,5,6,7,8$, and 9).

As a result, the resonance angle has the most variation for the sample 1 (after rotary evaporator), and the resonance angle shift for samples 5, 6, and 7 (after $6 \mathrm{~h}, 8 \mathrm{~h}$, and $10 \mathrm{~h}$ ) is zero. The concentration value of each sample was achieved from Figure 9(b), and the results were listed in Table 3.

The effect of palm kernel's moisture content on the concentration of n-hexane in the purification of oil was studied. As literature, the amount of moisture content has affected the amount of fatty acid and free fatty acids. In some process, the amount of $\mathrm{n}$-hexane and moisture content has an effect on the speed of reaction. Hence, the concentration of $n$-hexane was measured in the extracted oil from palm kernels with 


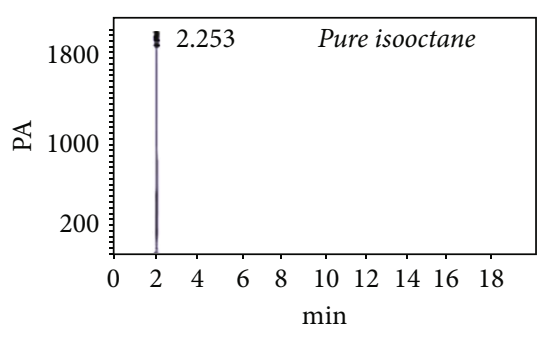

(a)

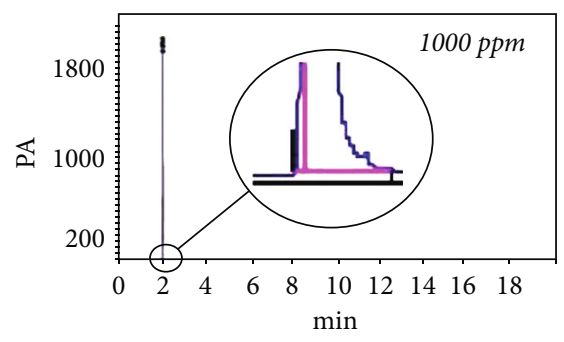

(c)

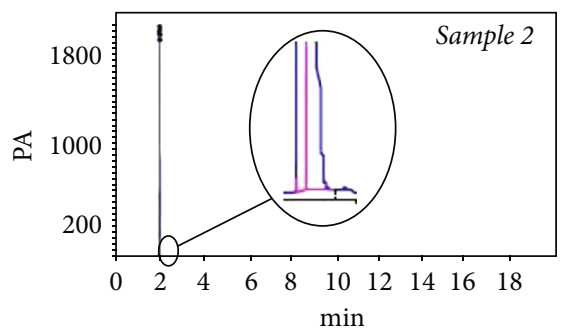

(e)

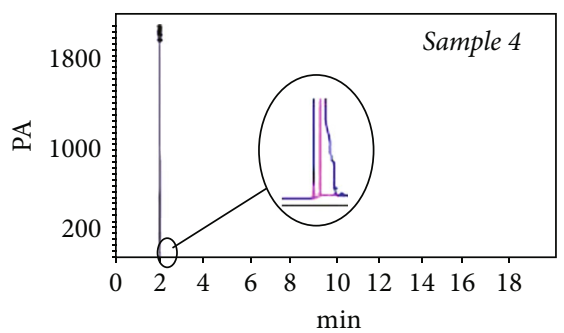

(g)

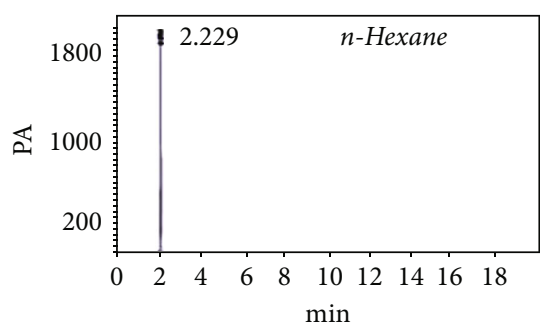

(b)

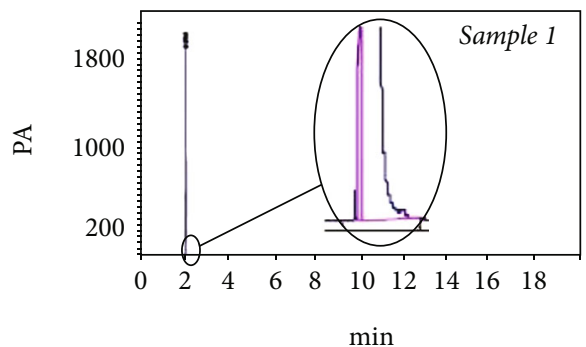

(d)

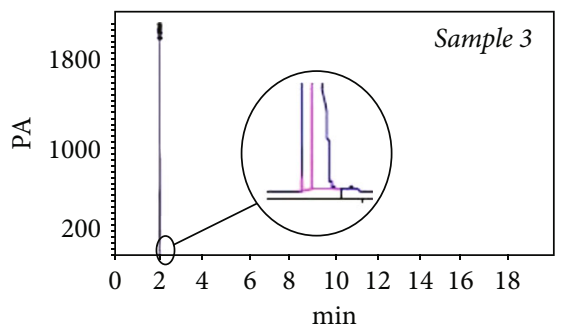

(f)

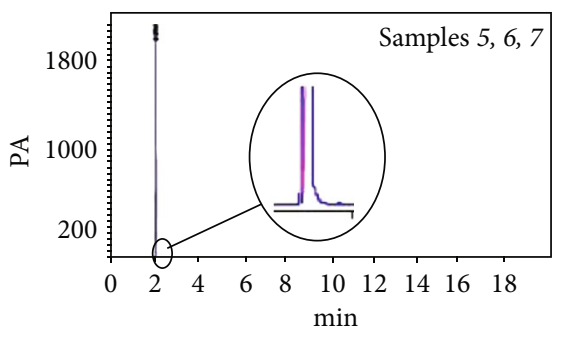

(h)

FIgURE 11: The GC results of (a) pure isooctane, the peak is at 2.253; (b) pure n-hexane, the peak is at 2.229; (c) the solution of n-hexane with 1000 ppm concentration; (d) sample 1 (rotary evaporator); (e) sample 2 (after 2 hours rotary and oven); (f) sample 3; (g) samples 4 and (h) 5 , 6,7 (after $6 \mathrm{~h}, 8 \mathrm{~h}$, and $10 \mathrm{~h}$ ).

$17 \%$ and $7 \%$ moisture content. Figure 10(a) shows the sensogram for measurement of the concentration of n-hexane in extracted oil from palm kernels with 7\% (see Figure 10(b)) and $17 \%$ (see Figure 10(c)) moisture contents. As a result, the concentration of n-hexane was 1573 and $1630 \mathrm{ppm}$ in the extracted oil from palm kernels with $17 \%$ and $7 \%$ moisture content, respectively. In other words, the concentration of n-hexane increased with decreasing the moisture content.

There are many methods such as nuclear magnetic resonance, gas chromatography, UV-visible, and Fourier transform infrared spectroscopy that can use to investigate and test the concentration of the organic solution. Among these methods, gas chromatography (GC) is a simple, affordable, reliable, and accurate method. Hence, to test the results, the samples were tested using GC. GC is fast and sensitive technique to recognize the volatile chemical material such as $\mathrm{n}$-hexane. Hence, the mixtures of isooctane and nhexane in concentration of 100,500,1000, and $5000 \mathrm{ppm}$; pure isooctane; and palm kernel oil (samples 1, 2, 3,4, 5, 6, and 7) were tested using gas chromatography (GC). The solutions were separately injected to the chromatography column. Figures 11(a)-11(h) show the GC results for pure n-hexane, pure isooctane, the solution of n-hexane in 1000 ppm concentration, the sample 1 (palm kernel oil after rotary evaporator), sample 2 (palm kernel oil after 2 hours rotary and oven), samples $3,4,5,6$, and 7 , respectively. The area under GC peak was obtained to find GC calibration curve. Figure 12 shows the calibration curve to evaluate the concentration of $\mathrm{n}$-hexane in isooctane. After that, the samples $1,2,3,4,5,6$, and 7 were tested using the 


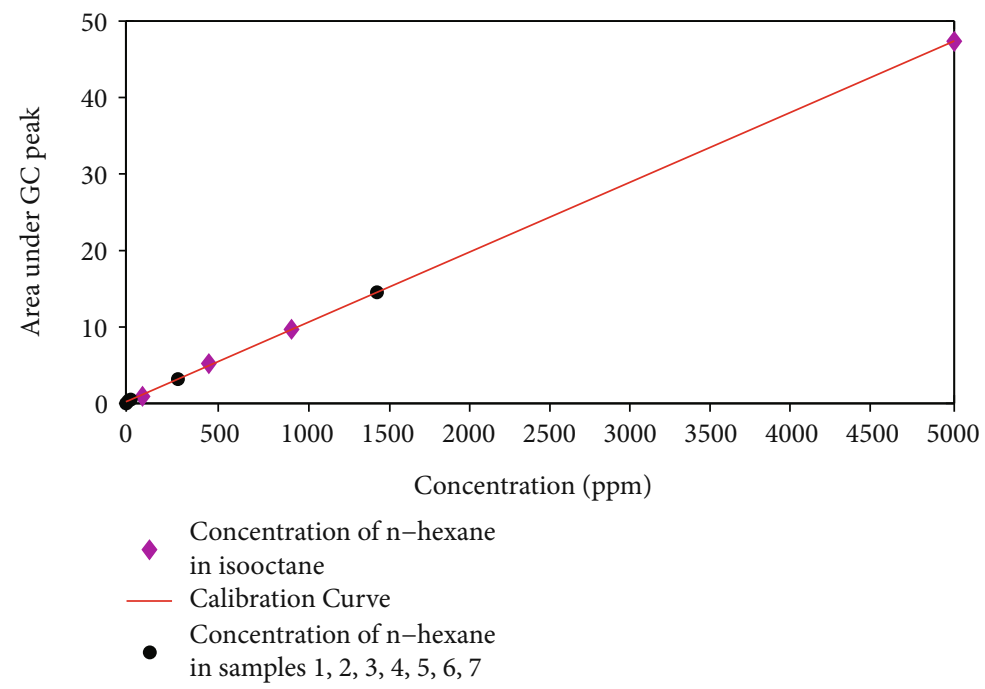

Figure 12: Calibration curve to obtain the concentration of n-hexane.

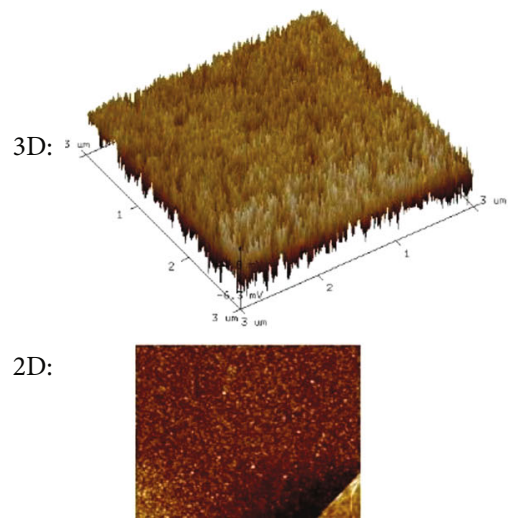

(a)
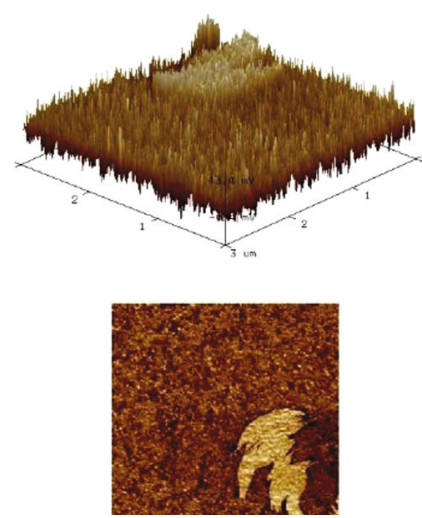

(b)

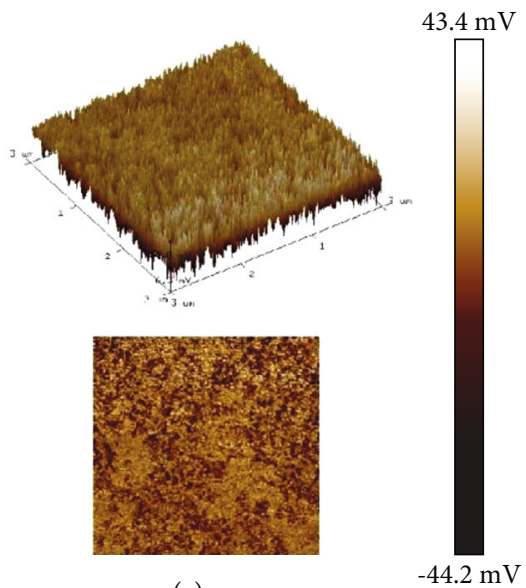

(c)

FIGURE 13: (a) The AFM image of sensing layer before contacting with $n$-hexane. (b) The AFM image of sensing layer after association process for detection of n-hexane. (c) The AFM image of sensing layer after dissociation process for detection of $n$-hexane.

chromatography method. The results were matched to the results that were achieved from SPR sensor. The results were listed in Table 3. As a result, the SPR sensor limit was $1 \mathrm{ppm}$ for detection of $n$-hexane, and it is higher than detection limit of other analytical methods such as GC. The SPR sensor can detect the low concentration of n-hexane, and the SPR sensor instrument is simpler than GC instrument. The SPR sensor is optical sensor, and it does not need special chemical knowledge and flowing gas.

In order to test the PPy-NPs/RGO as a sensing layer, the roughness of the layer was investigated before and after contacting with the prob medium. Figures 13(a)-13(c) show the atomic force microscopy images of sensing layer before detection of n-hexane, after association process, and after dissociation process, respectively. The images have depicted the interaction of n-hexane with PPy-NPs/RGO sensing layer. The morphology, roughness, and the thickness of the
TABLE 4: The pertinent parameter of AFM.

\begin{tabular}{lccc}
\hline Image & $\begin{array}{c}\text { Image surface area } \\
\left(\mu \mathrm{m}^{2}\right)\end{array}$ & $\begin{array}{c}\text { Thickness } \\
(\mathrm{nm})\end{array}$ & $\begin{array}{c}\text { Roughness } \\
(\mathrm{nm})\end{array}$ \\
\hline $\mathrm{a}$ & 9.14 & $10.3 \mathrm{~nm}$ & 6.56 \\
$\mathrm{~b}$ & 13.8 & $16.4 \mathrm{~nm}$ & 9.57 \\
$\mathrm{c}$ & 16.6 & $12.3 \mathrm{~nm}$ & 7.31 \\
\hline
\end{tabular}

PPy-NPs/RGO changed. The thickness and the roughness of the sensing layer increased from 10.3 to $16.4 \mathrm{~nm}$ and 6.56 to $9.57 \mathrm{~nm}$, respectively (Table 4 ).

Consequently, the $\mathrm{n}$-hexane can interact with the $\mathrm{PPy}$ NPs/RGO layer with the van der Waals bonding. So, it is the cause of variation of the resonance angle shift with time in association process (see Figures 6 and 9). 


\section{Conclusions}

The palm kernel oil was derived from extracting palm kernel using n-hexane. The FT-IR results confirmed that the n-hexane exited in the palm kernel oil before and after evaporation, and the difference between of samples before and after the evaporation in rotary evaporator and heating was $\mathrm{n}$-hexane content.

The PPy-NPs/RGO as a sensing layer has interacted with $\mathrm{n}$-hexane solution (n-hexane in isooctane) and palm kernel oil. Since the isooctane is a pure solvent, the resonance angle shift just depends on the concentration of $n$-hexane in the range of $1 \mathrm{ppm}$ to $10000 \mathrm{ppm}$. The refractive index of solution was measured using surface plasmon resonance technique in the range of 1.39094 to 1.38712 before contacting to sensing layer. The sensogram was obtained in the precancer of the sensing layer, and the limit of the sensor was about $1 \mathrm{ppm}$. The palm kernel oil solution was contacted to the sensing layer separately. The concentration of $n$-hexane in palm kernel oil was detected and measured in the range of 12 to $1516 \mathrm{ppm}$, and the results were matched with the concentrations of $n$-hexane which were obtained from gas chromatography. The thickness and roughness of the sensing layer surface were increased during the interaction of $n$ hexane with sensing layer that was achieved from an atomic force microscopy image. Consequently, the SPR sensor was applied to measure the low concentration of n-hexane, and the cols layer was modified using PPy-NPs/RGO nanocomposite layer. The PPy-NPs/RGO can interact with the n-hexane, and the sensor can detect the $\mathrm{n}$-hexane with $1 \mathrm{ppm}$ limit during $320 \mathrm{~s}$. As an AFM result, the roughness of the sensing layer was increased after the association process, and it decreased after the dissociation process. Consequently, the sensing layer was reversible.

\section{Data Availability}

The data used to support the findings of this study are available from the corresponding author upon request.

\section{Conflicts of Interest}

The authors declare that there are no conflicts of interest regarding the publication of this paper.

\section{Acknowledgments}

The second authors acknowledge support from the Universiti Putra Malaysia for $\mathrm{PhD}$ degree, and the authors acknowledge support from the Institute of Advanced Technology and Faculty of Science to provide the analytical facilities. The authors acknowledged from the Grant Nanomite (No. 5526307).

\section{Supplementary Materials}

Figure 1S: the SPR signals for PPy-NPs/RGO at temperature of (a) $20^{\circ} \mathrm{C}$, (b) $35^{\circ} \mathrm{C}$, (c) $31^{\circ} \mathrm{C}$, (d) $37^{\circ} \mathrm{C}$, and (e) variation of resonance angle with temperature. Figure $2 \mathrm{~S}$ : variation of resonance angle shift for pure palm oil. (Supplementary Materials)

\section{References}

[1] M. Y. Zulkafli, N. K. Othman, A. M. Lazim, and A. Jalar, "Inhibitive effects of palm kernel oil on carbon steel corrosion by alkaline solution," AIP Conference Proceedings, vol. 1571, pp. 42-47, 2103.

[2] T. P. Pantzaris and J. A. Mohd, "Properties and utilization of palm kernel oil," Palmoilis, vol. 35, pp. 11-23, 2001.

[3] D. K. Saxena, S. K. Sharma, and S. S. Sambi, "Comparative extraction of cottonseed oil by n-hexane and ethanol," Journal of Engineering and Applied Science, vol. 6, pp. 84-89, 2011.

[4] M. E. Barsan, NIOSH pocket guide to chemical hazards, National Institute for Occupational Safety and Health, 2007.

[5] D. S. Chadha, "Prevention of Food Adulteration Act. (PFA Act No.37 of 1954: Part III, Rule No.5, APPENDIX B Definition and Standards of Quality, A.17.02)".

[6] M. E. S. Mirghani and Y. B. Che Man, "Determination of hexane residues in vegetable oils with FTIR spectroscopy," Journal of the American Oil Chemists' Society, vol. 80, no. 7, pp. 619623, 2003.

[7] M. Yousefi and H. Hosseini, "Evaluation of hexane content in edible vegetable oils consumed in Iran," Journal of Experimental and Clinical Toxicology, vol. 1, p. 1, 2017.

[8] A. R. Sadrolhosseini, M. M. Moksin, M. M. Yunus, Z. A. Talib, and M. M. Abdi, "Surface plasmon resonance detection of copper corrosion in biodiesel using polypyrrole-chitosan layer sensor," Optical Review, vol. 18, no. 4, pp. 331-337, 2011.

[9] A. R. Sadrolhosseini, S. A. Rashid, A. S. M. Noor, H. N. Lim, Y. S. Lim, and M. A. Mahdi, "Reduced graphene oxide decorated with polypyrrole nanoparticles layer for detection of pyrene using surface plasmon resonance technique," ECS Journal of Solid State Science and Technology, vol. 5, pp. Q7-Q12, 2015.

[10] A. R. Sadrolhosseini, M. M. Moksin, M. M. Yunus, and Z. A. Talib, "Surface plasmon resonance determination of methanol concentration during alkaline transestrification," Journal of Engineering and Applied Science, vol. 9, pp. 54-57, 2010.

[11] J. Homola and P. Marek, Surface plasmon resonance based sensors, Springer, Berlin, Heidelberg, 2006.

[12] A. R. Sadrolhosseini, A. S. M. Noor, and M. M. Moksin, "Application of surface plasmon resonance based on a metal nanoparticle," in Plasmonics-Principles and Applications, K. Y. Kim, Ed., pp. 253-282, IntechOpen, 2012.

[13] S. Isaacs and I. Abdulhalim, "Long range surface plasmon resonance with ultra-high penetration depth for self-referenced sensing and ultra-low detection limit using diverging beam approach," Applied Physics Letters, vol. 106, no. 19, article 193701, 2015.

[14] L. Zhu, Z. Zhao, P. Cheng et al., "Antibody-mimetic peptoid nanosheet for label-free serum-based diagnosis of Alzheimer's disease," Advanced Materials, vol. 29, no. 30, article 1700057, 2017.

[15] H. Ma, J. He, X. Liu, J. Gan, G. Jin, and J. Zhou, "Surface initiated polymerization from substrates of low initiator density and its applications in biosensors," ACS Applied Materials \& Interfaces, vol. 2, no. 11, pp. 3223-3230, 2010.

[16] B. Wu, Q. W. Jiang, Q. Wang et al., "Detection of C-reactive protein using nanoparticle-enhanced surface plasmon resonance using an aptamer-antibody sandwich assay," Chemical Communications, vol. 52, no. 17, pp. 3568-3571, 2016. 
[17] R. Mamta, G. Pankaj, N. G. Rajendra, and S. Yoon-Bo, "Graphene/conducting polymer nano-composite loaded screen printed carbon sensor for simultaneous determination of dopamine and5-hydroxytryptamine," Sensors and Actuators B: Chemical, vol. 239, pp. 993-1002, 2017.

[18] Y. Liang, L. Yu, R. Yang, X. Li, L. Qu, and J. Li, "High sensitive and selective graphene oxide/molecularly imprinted polymer electrochemical sensor for 2,4-dichlorophenol in water," Sensors and Actuators B: Chemical, vol. 240, pp. 1330-1335, 2017.

[19] Y. Zhou, Z.-b. Qu, Y. Zeng, T. Zhou, and G. Shi, "A novel composite of graphene quantum dots and molecularly imprinted polymer for fluorescent detection of paranitrophenol," Biosensors \& Bioelectronics, vol. 52, pp. 317-323, 2014.

[20] W. Zheng, M. Zhao, W. Liu et al., "Electrochemical sensor based on molecularly imprinted polymer/reduced graphene oxide composite for simultaneous determination of uric acid and tyrosine," Journal of Electroanalytical Chemistry, vol. 813, pp. 75-82, 2018.

[21] X. Zhou, P. Ma, A. Wang et al., "Dopamine fluorescent sensors based on polypyrrole/graphene quantum dots core/shell hybrids," Biosensors and Bioelectronics, vol. 64, pp. 404-410, 2015.

[22] A. R. Sadrolhosseini, S. Abdul Rashid, N. Jamaludin, A. S. M. Noor, and A. M. Isloor, "Surface plasmon resonance sensor using polypyrrole-chitosan/graphene quantum dots layer for detection of sugar," Materials Research Express, vol. 6, no. 7, 2019.

[23] Y.-C. Liu and C.-J. Tsai, "Enhancements in conductivity and thermal and conductive stabilities of electropolymerized polypyrrole with caprolactam-modified clay," Chemistry of Materials, vol. 15, no. 1, pp. 320-326, 2003.

[24] B. R. Saunders, R. S. Fleming, and K. S. Murray, "Recent advances in the physical and spectroscopic properties of polypyrrole films, particularly those containing transition-metal complexes as counteranions," Chemistry of Materials, vol. 7, no. 6, pp. 1082-1094, 1995.

[25] N. Gupta, S. Sharma, I. A. Mir, and D. J. Kumar, "Advances in sensors based on conducting polymers," Journal of Scientific and Industrial Research, vol. 65, pp. 549-557, 2006.

[26] A. M. Dimiev and J. Tour, "Mechanism of graphene oxide formation,” ACS Nano, vol. 8, no. 3, pp. 3060-3068, 2014.

[27] D. S. Sutar, G. Singh, and V. Divakar Botcha, "Electronic structure of graphene oxide and reduced graphene oxide monolayers," Applied Physics Letters, vol. 101, no. 10, p. 103103, 2012.

[28] L. Wu, H. S. Chu, W. S. Koh, and E. P. Li, "Highly sensitive graphene biosensors based on surface plasmon resonance," Optics Express, vol. 18, no. 14, pp. 14395-14400, 2010.

[29] S. Muhammad Hafiz, R. Ritikos, T. J. Whitcher et al., "A practical carbon dioxide gas sensor using room-temperature hydrogen plasma reduced graphene oxide," Sensors and Actuators B: Chemical, vol. 193, pp. 692-700, 2014.

[30] E. Hendry, P. J. Hale, J. Moger, A. K. Savchenko, and S. A. Mikhailov, "Coherent nonlinear optical response of graphene," Physical Review Letters, vol. 105, no. 9, article 097401, 2010.

[31] M. B. Hisyam, M. F. M. Rusdi, A. A. Latiff, and S. W. Harun, "Generation of mode-locked ytterbium doped fiber ring laser using few-layer black phosphorus as a saturable absorber," IEEE Journal of Selected Topics in Quantum Electronics, vol. 23, no. 1, pp. 39-43, 2017.
[32] Y. Xu, Y. S. Ang, L. Wu, and L. K. Ang, "High sensitivity surface plasmon resonance sensor based on two-dimensional MXene and transition metal dichalcogenide: a theoretical study," Nanomaterials, vol. 9, no. 2, p. 165, 2019.

[33] H. Liu, L. Zhang, Y. Guo et al., "Reduction of graphene oxide to highly conductive graphene by Lawesson's reagent and its electrical applications," Journal of Materials Chemistry C, vol. 1, no. 18, pp. 3104-3109, 2013.

[34] W. H. Macaire, T. Bernard, T. Noel et al., "Extraction of palm kernel oil in Cameroon: effects of kernels drying on the oil quality," Journal of Food Technology, vol. 8, no. 1, pp. 1-7, 2010.

[35] A. R. Sadrolhosseini, S. A. Rashid, A. S. M. Noor, A. Kharazmi, H. N. Lim, and M. A. Mahdi, "Optical band gap and thermal diffusivity of polypyrrole-nanoparticles decorated reduced graphene oxide nanocomposite layer," Journal of Nanomaterials, vol. 2014, 2016.

[36] N. M. Huang, H. N. Lim, C. H. Chia, M. A. Yarmo, and M. R. Muhamad, "Simple room-temperature preparation of highyield large-area graphene oxide," International Journal of Nanomedicine, vol. 6, article 3443, 2011.

[37] A. R. Sadrolhosseini, M. Naseri, and S. A. Rashid, "Polypyrrole-chitosan/nickel-ferrite nanoparticle composite layer for detecting heavy metal ions using surface plasmon resonance technique," Optics and Laser Technology, vol. 93, pp. 216223, 2017.

[38] A. R. Sadrolhosseini, M. Naseri, and H. M. Kamari, "Surface plasmon resonance sensor for detecting of arsenic in aqueous solution using polypyrrole-chitosan-cobalt ferrite nanoparticles composite layer," Optics Communication, vol. 383, pp. 132-137, 2017.

[39] N. Hari and V. P. Nair, "FTIR spectroscopic analysis of leaf extract in Hexane in Jasminum Azoricum L," Recent Research in Science and Technology, vol. 4, pp. 170-172, 2018.

[40] C. F. Uzoh, N. J. Obodo, and O. D. Onukwuli, "Exploring the effect of styrene and anhydride ratio on the coating properties of non-drying vegetable oil based alkyd resin," Journal of King Saud University - Engineering Sciences, vol. 30, no. 1, pp. 1221, 2018.

[41] H. Jahangirian, M. J. Haron, N. A. Yusof et al., "Enzymatic synthesis of fatty hydroxamic acid derivatives based on palm kernel oil," Molecules, vol. 16, no. 8, pp. 6634-6644, 2011.

[42] M. Naseri, A. R. Sadrolhosseini, Y. W. Fen, and M. A. Mahdi, "Measurement of low magnesium concentration in aqueous solution," Sensors and Materials, vol. 30, pp. 1019-1026, 2018. 